Breathing therapies in asthma

\section{Breathing therapies and bronchodilator use in asthma}

\author{
A Bruton, M Thomas
}

\section{Breathing modification techniques may have a useful role in the management of asthma}

$\mathrm{T}$ reatments involving the manipulation of breathing are commonly used in routine practice by physiotherapists and other practitioners in a variety of clinical settings ${ }^{1}$ yet, despite decades of use, there is no robust evidence base to confirm the effectiveness of these activities. Practical and logistic difficulties exist in the design and execution of the randomised controlled clinical trials required to provide the highest grade evidence for such treatments. While the design of studies investigating pharmacological agents is relatively straightforward, there are numerous potential pitfalls in trials investigating complex interventions such as breathing therapies which require significant interaction between patient and practitioner. There are obvious difficulties with devising suitable controls, blinding participants and researchers to any intervention received, and in ensuring standardisation. While it is frequently clinically acceptable to standardise medication dosage and timing, it is not normal to do so for breathing therapies-in which the response of the individual affects the intervention provided during any interaction between patient and practitioner.

\section{STUDY OF BREATHING TECHNIQUES}

In this issue of Thorax, Slader et al present the results of a randomised double blind controlled trial comparing two breathing techniques for patients with stable but suboptimally controlled asthma. Fifty seven subjects were randomly allocated to one of two groups receiving instruction in breathing technique from educational videos, and 48 subjects completed the 30 week study. The first group of 28 subjects were instructed in a technique designed to be "active" by mimicking the hypoventilation and nasal breathing strategies advocated by the exponents of the Butekyo breathing method, and the second group of 29 subjects received an instructional video that was intended to be a more neutral control, consisting of upper chest exercises and "control of breathing" exercises focusing on posture and relaxation. Subjects were instructed to practise the exercises twice daily and as needed for relief of symptoms. Strictly speaking, this was a negative study in that no clinically important differences were observed between the groups following the interventions; however, a marked reduction in the use of short acting $\beta_{2}$ agonist bronchodilator medication was seen in both groups compared with the baseline period, without significant changes in lung function, airways hyperreactivity, or end tidal $\mathrm{CO}_{2}$. Although there were no differences between groups for changes in patient centred outcomes such as quality of life and Asthma Control Questionnaire scores, consistent trends were seen with improvements in both groups over the baseline period. These improvements were maintained over 28 weeks in spite of a second phase of back-titration of inhaled corticosteroids which achieved a 50\% reduction in dose that was similar in both groups. Although a reduction in inhaled corticosteroid dose without a loss of control is welcome news, as the authors state it cannot be assumed that this is a result of the breathing exercises; it is recognised that many patients with asthma are overtreated and recent step-down studies have reported similar dose reduction without the use of any intervention. ${ }^{3}$

Of more interest is the reduction in bronchodilator use. Previous trials involving breathing therapies for asthma medication usage, although not of this magnitude. If individuals were able to reduce their bronchodilator usage by the amount reported $(86 \%)$ without any accompanying deterioration in their asthma control, this is worthy of comment. The authors have suggested some possible mechanisms for this, including the possibility that the two breathing routines provided a non-specific deferral strategy for $\beta_{2}$ agonist use.

This study raises important questions about the design of trials investigating the effectiveness of breathing therapies, have also reported a reduction in rescue and on strategies for reducing bronchodilator use without compromising asthma outcomes.

\section{PROBLEMS OF TRIAL DESIGN}

Designing any convincing yet inert placebo intervention for breathing therapy is extremely challenging. Previous authors have used controls involving other breathing manoeuvres, relaxation, education, videos, and sham training devices. ${ }^{4-7}$ There are problems with nearly all of these. We currently have minimal understanding of the physiological or psychological effects of any of the respiratory manoeuvres used in clinical practice. ${ }^{8}$ Relaxation techniques themselves invariably involve some modulation of breathing pattern, and "breathing control" will also incorporate relaxation techniques. ${ }^{9}$ Slader et al have addressed the issues of standardisation and patient-practitioner interaction by providing both the intervention and control technique via a video. Unfortunately, this results in a rather simplistic approach to a fairly complex intervention, as to teach any form of exercise effectively requires not only initial demonstration and subsequent practice but also feedback on individual performance to ensure conformity with the prescribed exercise. Although videos have been used in previous trials of breathing techniques, there is some evidence that viewing a television screen in itself may have a relaxing effect and alter breathing patterns in comparison with other sedentary activities such as reading. ${ }^{10}$

In the study by Slader et al, a supposedly active intervention involving "hypoventilation", "breath holding", and "nasal breathing" - a package that closely mimics the main elements of the Butekyo breathing technique ${ }^{11}$-is compared with a control involving "nonspecific upper body exercises". However, on closer examination the latter included "controlled inspiratory-expiratory cycles" during arm exercises as well as "control of breathing" and "relaxation". This means that both arms of the trial involved altering breathing patterns, although—as is common with most trials of breathing therapy-the interventions are not described in sufficient detail to permit accurate replication. Terms like "control of breathing" can be interpreted in many ways, but techniques generally involve asking people to breathe at a slower than normal rate and/or at reduced volumes and flows. Unfortunately, this suggests manipulation of breathing patterns in a way similar to many active therapies, and this control intervention may not have been inert. It is therefore possible that the similar reduction in bronchodilator use seen in the two study arms 
may have resulted from two equally effective therapies, rather than indicating a lack of effect in the "active" treatment group.

Interventions to alter patterns of breathing are most likely to work in patients in whom the underlying breathing pattern is in some way abnormal Direct evidence for clinically significant altered patterns of breathing in asthma patients is not available, although some studies have indicated that patients with stable asthma are hypocapnic. ${ }^{12} 13$ Obtaining direct evidence of dysfunctional breathing has historically been very difficult, as use of mouthpieces/ masks inevitably affects breathing patterns, and observations in a laboratory setting may induce anxiety leading to hyperventilation in some patients. ${ }^{14}$ Indirect evidence comes from surveys of asthma patients reporting up to $30 \%$ prevalence of "dysfunctional breathing" when assessed using the Nijmegen hyperventilation questionnaire. ${ }^{15} \mathrm{~A}$ small trial of physiotherapy breathing retraining targeting such patients showed clinically significant improvements in quality of life in over half the subjects. ${ }^{4}$ Recent technological improvements are currently making non-invasive ambulatory measurement during daily activity into a practical possibility. ${ }^{17}$ This should provide us with more information about patterns of breathing in asthma and enable us to identify those with dysfunctional breathing. Future trials of breathing therapies could then be targeted specifically at this group. The same technology has the potential to provide baseline and outcome measures for breathing therapies to establish whether any genuine change in breathing pattern has occurred.

\section{REDUCED USE OF $\beta_{2}$ AGONISTS}

An observed reduction in $\beta_{2}$ agonist use of almost $90 \%$ is of interest, particularly at a time when the safety profile of $\beta$ agonists has once again come under the spotlight. ${ }^{18}$ There have been concerns for some time that high levels of use of short acting $\beta_{2}$ agonists may be associated with an increased risk of severe asthma attacks and death, ${ }^{19}$ although it remains uncertain as to whether this represents a pharmacological risk or acts as a marker for more severe and uncontrolled asthma. ${ }^{20}$ There is evidence that frequent administration of short acting $\beta_{2}$ agonists results in some loss of bronchodilation ${ }^{21-23}$ and a decrease in bronchoprotective action, ${ }^{24}$ particularly in patients with certain genotypes of the $\beta_{2}$ adrenergic receptor. ${ }^{25}$ In addition, discontinuation of frequent $\beta$ agonist use may result in a transient rebound decrease in pulmonary function and increase in bronchial hyperresponsiveness. ${ }^{23}$ Regular exposure to inhaled $\beta$ agonists may have detrimental cardiac effects, particularly on those with pre-existing cardiac disease. ${ }^{26}$ There is therefore a good case to be made for any intervention, particularly a nonpharmacological one, which can reduce rescue bronchodilator use without compromising asthma control.

Was the reduction in bronchodilator use a result of the breathing exercises in the two arms of the study or was it due to other non-specific mechanisms? The subjects recruited to this study were clinically stable but had a relatively high bronchodilator use at baseline ( 3 puffs per day) so "regression to the mean" and trial involvement effects may explain some of the reduction, although the magnitude of the reduction and the rapidity of the effect which commenced immediately after the beginning of the intervention and continued to improve over the initial 12 week study phase make it unlikely this could be a full explanation. Subjects in both groups were instructed when symptomatic to use their breathing exercises for up to 5 minutes before using rescue medication, so it is possible that the medication reduction resulted from a short deferment of use of rescue bronchodilator and allowed spontaneous resolution of symptoms, rather than from a specific effect of the exercises. If this were the case, a viable deferment strategy could be applicable to many asthmatics, and giving instructions on deferment of bronchodilator use plus provision of an acceptable alternative action such as a breathing exercise (even if this is physiologically inert) may be valuable. Further studies are needed to confirm the reliability and generalisability of these findings, which potentially have considerable significance for asthma management, and to investigate the mechanisms of apparent effectiveness of these two breathing exercise programmes.

This study poses more questions than it answers, but does again raise the possibility that breathing modification techniques may have a useful role to play in the management of asthmawhich patients may be helped, which techniques are most effective, and by what mechanism of action still remain to be clarified.

\section{Thorax 2006;61:643-645.}

doi: $10.1136 /$ thx.2005.057422

\section{Authors' affiliations}

A Bruton, University of Southampton, Southampton, UK

M Thomas, Department of General Practice, University of Aberdeen, Aberdeen, UK

Correspondence to: Dr M Thomas, Cotswold Cottage, Oakridge, Stroud, Gloucestershire GL6 7NZ, UK; mikethomas@doctors.org.uk
Competing interests: none declared.

\section{REFERENCES}

1 Prasad NH, Webber BA, Pryor JA, et al. Physiotherapy for respiratory and cardiac problems: adults and paediatrics. Edinburgh: Churchill Livingstone, 2004.

2 Slader CA, Reddel HK, Spencer LM, et al. Double blind randomised controlled trial of two different breathing techniques in the management of asthma. Thorax 2006:61:651-6.

3 Hawkins G, McMahon AD, Twaddle S, et al. Stepping down inhaled corticosteroids in asthma: randomised controlled trial. BMJ 2003;326:1115.

4 Thomas M, McKinley RK, Freeman E, et al. Breathing retraining for dysfunctional breathing in asthma- a randomised controlled trial. Thorax 2003;58: $110-5$

5 Bowler SD, Green A, Mitchell CA. Butekyo breathing techniques in asthma: a blinded randomised controlled trial. Med J Aust 1998; 169:575-8.

6 Opat AJ, Cohen MM, Bailey MJ, et al. A clinical trial of the Buteyko breathing technique in asthma as taught by a video. J Asthma 2000;37:557-64.

7 Cooper S, Oborne J, Nelson S, et al. The effect of two breathing exercises (Butekyo and pranayama) in asthma: a randomised controlled trial. Thorax 2003;58:674-9.

8 Bruton A, Clark R. A pilot study measuring mixed venous carbon dioxide levels in students with and without a diagnosis of asthma. Physiotherapy 2004;90:120-4.

9 Pryor JA, Pryor JA. Physiotherapy for respiratory and cardiac problems. Edinburgh: Churchill Livingstone, 1998.

10 Hark WT, Thompson WM, McLaughlin TE, et al. Spontaneous sigh rates during sedentary activity: watching television vs reading. Ann Allergy Asthma Immunol 2005;94:247-50.

11 Stalmatski A. Freedom from asthma: Butekyo's revolutionary treatment. London: Kyle Cathie Ltd, 1997

12 Osborne CA, O'Connor BJ, Lewis A, et al. Hyperventilation and asymptomatic chronic asthma. Thorax 2000;55:1016-22.

13 van den Elshout FJ, van Herwaarden $\mathrm{CL}$ Folgering HT. Effects of hypercapnia and hypocapnia on respiratory resistance in norma and asthmatic subjects. Thorax 1991;46:28-32.

14 Askanazi J, Silverberg PA, Foster RJ, et al. Effects of respiratory apparatus on breathing pattern. $J$ Appl Physiol 1980;38:577-80.

15 Martinez-Moragon E, Perpina M, Belloch A, et al. Prevalence of hyperventilation syndrome in patients treated for asthma in a pulmonology clinic. Arch Bronconeumol 2005;41:267-71.

16 Thomas M, McKinley RK, Freeman E, et al. Prevalence of dysfunctional breathing in patients treated for asthma in primary care: a cross-sectional survey. BMJ 2001;322:1098-100.

17 Wilhelm FH, Roth W, Sackner MA. The LifeShirt. An advanced system for ambulatory measurement of respiratory and cardiac function. Behav Modif 2003;27:671-91.

18 Nelson HS. Is there a problem with inhaled longacting $\beta$-adrenergic agonists? J Allergy Clin Immunol 2006; 117:3-16.

19 Spitzer WO, Suissa S, Ernst P, et al. The use of beta-agonists and the risk of death and near death from asthma. N Engl J Med 1992;326:501-6.

20 Blais L, Ernst $P$, Suissa $S$. Confounding by indication and channeling over time: the risks of beta2-agonists. Am J Epidemiol 1996; 144:1169.

21 Cockcroft D, McParland CP, Britto SA, et al. Regular inhaled salbutamol and airway responsiveness to allergen. Lancet 1993;342:833-7.

22 Inman MD, O'Byrne P. The effect of regular inhaled albuterol on exercise-induced bronchoconstriction. Am J Respir Crit Care Med 1996;153:65-9. 
23 Vathenen AS, Knox AJ, Higgins BG, et al Rebound increase in bronchial responsiveness after treatment with inhaled terbutaline. Lancet 1988; 1:554-8

24 Bhagat R, Swystun VA, Cockcroft DW. Salbutamol-induced increased airways responsiveness to allergen and reduced protection versus methacholine: dose response. J Allergy Clin Immunol 1996:97:47-52.

25 Israel E, Drazen JM, Liggett SB, et al. The effect of polymorphisms of the $\beta_{2}$ adrenergic receptor on the response to regular use of albuterol in asthma. Am J Respir Crit Care Med 2000; 162:75-80.

26 Suissa S, Hemmelgard B, Blais L, et al. Bronchodilators and acute cardiac death. Am J Respir Crit Care Med 1996;154:1598-602.

\section{Pseudomonas serology: confusion, controversy, and challenges}

\section{P M Farrell, J R W Govan}

\section{Contrasting messages on the diagnostic value of Pseudomonas serology in CF}

$\mathrm{T}$ he two interesting but contrasting (and possibly confusing) papers on Pseudomonas serology in cystic fibrosis (CF) published in this issue of Thorax illustrate the controversy and challenge that have become increasingly important as very young patients are routinely diagnosed through newborn screening. Fortunately, such infants at diagnosis are typically free of Pseudomonas aeruginosa (PA) infection, ${ }^{1}$ unlike about 30\% of those diagnosed by traditional methods following signs/symptoms of CF. The potential to eradicate non-mucoid $\mathrm{PA}$, and even to delay transformation to mucoid species, ${ }^{1}$ makes ascertainment of the initial PA infection one of the highest priorities in current clinical management. Yet, just as in the diagnosis of CF per se, traditional methods of PA identification (relying on microbiology) leave much to be desired in young children with CF. Thus, more attention has focused once again on the potential diagnostic value of Pseudomonas serology. In a recent review Rosenfeld et $a l^{3}$ stated that "limitations of serologic markers of $P$ aeruginosa infection include lack of commercially available standardized assays and lack of specificity to the site of $P$ aeruginosa infection (i.e. upper or lower airway)".

The pioneering studies on Pseudomonas serology were published 23 decades ago when the research teams of Niels Hoiby and Gerd Doring developed the initial methodology and applied Pseudomonas antibody titre determinations to patients with $\mathrm{CF}^{4-6}$ The first tests were based on detection of "precipitating antibodies" (precipitins) against a pool of sonicated extracts from common 0 -antigen serotypes. Their evaluations showed that rising antibody titres correlated with PA respiratory infections. They also showed $^{6}$ that, soon after the onset of PA lung infections, the numbers of individual precipitin bands rose and eventually became a sign of "poor prognosis". Subsequently, Brett and co-workers $^{7}$ published important research describing the rising antibody titres and potential for early identification of PA infections. More recent investigations ${ }^{18}$ have confirmed the findings of Brett et $a l^{7}$ and support the potential usefulness of Pseudomonas serology in children with CF.

When viewed against this background, the investigations of Kappler et $a l^{9}$ and Tramper-Stranders et a ${ }^{10}$ are significant steps forward, although their contrasting conclusions appear to represent steps in different directions. Our independent reviews of the papers led both of us to recommend that they be published with revisions because we were favourably impressed with the quality of the studies. Not knowing the identity of the other reviewer until commissioned to write this editorial, and judging each manuscript on its own merits, we reached similar decisions for a variety of reasons and appreciate having this opportunity to share our perspectives. However, distinguishing the two studies during the review process was a challenge for each of us. Hopefully, this commentary will alleviate confusion, explain why controversies are currently inevitable regarding Pseudomonas serology, and highlight some of the future challenges. We begin with a summary of the articles from Munich and Utrecht, as outlined in table 1 .

It is important to emphasise that both studies used the same commercially available ELISA test system, but different cut off values were used to define a positive antibody titre. Specifically, the investigation in Munich by Kappler et a ${ }^{9}$ simply used the manufacturer's recommended threshold (>1:500) with concomitant quality control mechanisms. In contrast, the investigation in Utrecht by Tramper-Stranders et al ${ }^{10}$ applied lower titres to discriminate PA antibody positivity after creating receiver-operator curves (ROC) to identify the titre cut off value that maximised sensitivity while preserving specificity. The ROC technique was developed originally in the 1940s to improve operator vigilance for radar based detection of incoming aeroplanes ${ }^{11}$ and applied ${ }^{12}$ successfully to "signal detectability and medical decision-making" in the 1970s. It is a valuable method but depends on having a reliable "gold standard" marker-for example, an actually observed aeroplane or a positive PA culture associated with lower respiratory infections. As described below, this requirement is a particular challenge with respiratory secretion cultures involving children. In fact, the "gold standard" is tarnished in this situation, and the usually valid assumption that sensitivity is a function of the test and not prevalence may not apply when an unreliable indicator exists- that is, oropharyngeal culture results. The design differences may also partially explain the discrepancy in conclusions. The Munich team had the advantage of repeated measures every 3 months in a prospective assessment. On the other hand, the Utrecht group actually performed two assessments (table 1): (A) a cross sectional evaluation of serological data compared with microbiological results, and (B) a prospective annual examination of the same variables in 4-14 year old children. Although the microbiologically determined PA status appeared somewhat similar, the methods of obtaining respiratory secretions necessarily varied among patients as shown clearly in table 1 in the paper by Kappler et al. ${ }^{9}$ Of the 183 patients, only $76(42 \%)$ expectorated sputum and thus oropharyngeal swabs were used for 107 patients. Tramper-Stranders et al ${ }^{10}$ state that "the sensitivity and positive predictive value of oropharyngeal swabs range between $44 \%$ and $83 \% "$, which reflects the results of a variety of studies using concurrent bronchoalveolar lavage and oropharyngeal swabs, as reviewed by TramperStranders et al. ${ }^{13}$ Table 1 in the paper by Kappler et $a l^{9}$ also exposes another 